Agriculture, Agribusiness and Biotechnology

Vol.59: e16160548, January-December 2016 http://dx.doi.org/10.1590/1678-4324-2016160548 ISSN 1678-4324 Online Edition

\title{
Establishment of an Embryonic Tip Regeneration System of Soybean
}

\author{
LIU Si-yan ${ }^{1 *}$, LI Guang-long ${ }^{1}$, QU Jing ${ }^{2}$, GUAN Shu-yan ${ }^{1}$, YAO Dan ${ }^{1}$, WANG Pi-wu ${ }^{2}$, LIU \\ Si-chen ${ }^{1}$, JIA Yang ${ }^{1}$, GUAN Peng-fei ${ }^{1}$, WANG Long-qing ${ }^{1}$. \\ ${ }^{1}$ College of Life Sciences, Jilin Agricultural University, Changchun, China; ${ }^{2}$ College of Agronomy, Jilin \\ Agricultural University, Changchun, China
}

\begin{abstract}
In order to establish an optimal soybean embryonic tip regeneration system that can serve as soybean genetic transformation receptor, and be used for the study of genetic function verification, the influences of single factor on the adventitious bud of embryonic tip induction, elongation and rooting stage, are researched and compared.The single factors includes seeds soaking time, different kinds of hormones, different concentration of hormone and different concentration of sucrose. By one-way ANOVA and LSD ad hoc test, the results show that, for the embryonic tip adventitious bud induction stage, $12 \mathrm{~h}$ is the optimal seeds soaking time, 2.0mg. $L^{-1}$ is the optimal concentration of 6-Benzyl Aminopurine(6-BA), for the embryonic tip adventitious bud elongation stage, $0.2 \mathrm{mg} \cdot \mathrm{L}^{-1}$ indole-3-butyric acid (IBA) is optimal and $2.0 \mathrm{mg} \cdot \mathrm{L}^{-1}$ Gibberellic acid $\left(G A_{3}\right)$ is optimal, and for the adventitious bud of embryonic tip rooting stage, $2.0 \mathrm{mg} \cdot \mathrm{L}^{-1}$ IBA is optimal, the average rooting rate is $93.34 \%$. An Optimal embryonic tip regeneration system is established, and optimum mediums in different stages are found.
\end{abstract}

Key words: Soybean; Induction; Elongation; Rooting; Single factor

\footnotetext{
*Authors for correspondence:siyan_2001@163.com
} 


\section{INTRODUCTION}

Soybean is a kind of annual herbaceous plants of Glycine Willd. It is native to China. Soybean is an important oil crop, so to improve the quality of soybean is an important goal of the modern breeding technique. A highly efficient and stable regeneration system is the foundation to achieving this goal. Finding a reasonable and productive way to improve the yield of soybean is particularly important ${ }^{[1]}$.

Tissue culture is considered to be a feasible way to increase soybean production. It is applied in soybean from the 1960s, but its disadvantage in low regeneration rate, poor reproducibility, genotype dependent, complex culture conditions, long regeneration $\mathrm{cycle}^{[2]}$ constrained the development of soybean tissue culture. Until to the $1980 \mathrm{~s}^{[3]}$, cotyledon nodes ${ }^{[4-8]}$, embryonic tips ${ }^{[9-11]}$, hypocotyls ${ }^{[12-14]}$ and protoplasts ${ }^{[15]}$ are used as explants, and soybean plants are successfully regenerated by tissue culture.

Embryonic tip regeneration system is a new soybean tissue culture regeneration system proposed in $2004^{[9]}$. It has some advantages in fast sprouting, neat growth, easy elongation, short growth cycle and good repeatability ${ }^{[16-19]}$. We use Jinong 18 soybean's embryonic tip as explants, research and compare the effects of different seeds soaking time, different kinds of hormones, different concentration of hormone and sucrose in the adventitious bud of embryonic tip induction, elongation and rooting stage. By the experiments, we hope to define optimal conditions for the embryonic tip regeneration system, and lay the foundation for the research of soybean genetic transformation.

\section{MATERIALS AND METHODS}

\subsection{Plant materials and seed disinfection}

We chosen Jinong 18, a soybean variety provided by the Plant Biotechnology Centre of Jilin Agricultural University, as the plant materials.

The healthy and plump soybean seeds were soaked in $70 \%$ ethanol for 30 s, and then were sterilized by chlorine for $16 \mathrm{~h}$. After the sterilization, we rinsed the seeds for five times with sterilized water.

2.2 Induction on adventitious buds of embryonic tip

\subsubsection{Effects of soaking time}

The seeds were divided to five groups, and soaked respectively with sterilized water for $6 \mathrm{~h}, 12 \mathrm{~h}, 18 \mathrm{~h}$,
$24 \mathrm{~h}$ and $36 \mathrm{~h}$. After the soak, peeled the seed coats, leaves and cotyledons, andisolated the hypocotyls and growing point ${ }^{[16]}$. Adventitious buds induction media was based on MS medium added with 2.0 mg.L ${ }^{-1}$ 6-BA. For optimum seeds soaking time, the replications of the treatment were done two times with five repetitions. Each repetition included four explants. The explants were planted for $10 \mathrm{~d}$ with lighting condition. After 10d, average induction rate of adventitious buds was analyzed by one-way ANOVA and LSD ad hoc test with software SPSS16.0.

\subsubsection{Effects of different concentrations of 6-BA} The seeds were soaked with sterilized water for 12h. After the soak, peeled seed coats, leaves and cotyledons, and isolated the hypocotyls and growing point. Adventitious buds induction media were based on MS medium added different concentration of 6-BA (Table 1). For optimum adventitious bud of embryonic tip induction media, the replications of the treatment were done two times with five repetitions. Each repetition used four explants. Explants were planted for 10d with light condition. After 10d, average induction rate of adventitious buds was analyzed by one-way ANOVA and LSD ad hoc test with software SPSS16.0.

Table 1.Media of adventitious buds induction treatment

\begin{tabular}{ll}
\hline Code & $6-\mathrm{BA}\left(\mathrm{mg} \cdot \mathrm{L}^{-1}\right)$ \\
\hline MI1 & 0 \\
MI2 & 1 \\
MI3 & 2 \\
MI4 & 3 \\
MI5 & 4 \\
\hline
\end{tabular}

2.3 Effects of different concentrations of hormone on elongation

Adventitious buds elongation media were based on MS medium added different concentration of 6BA, IBA and $\mathrm{GA}_{3}$ (Table2). For optimum adventitious bud of embryonic tip elongation media, the replications of the treatment were done two times with five repetitions. Each repetition included four explants. The explants were planted for $20 \mathrm{~d}$ with lighting condition. After $20 \mathrm{~d}$, average elongation rate of adventitious buds ( elongation rate $=$ the numbers of elongation buds ( the length is greater than $2 \mathrm{~cm}$ ) / the total numbers of 
explants $\times 100 \%$ ) was analyzed by one-way

SPSS16.0.

ANOVA and LSD ad hoc test with software

Table 2.Media of adventitious buds elongation treatment

\begin{tabular}{llll}
\hline Code & 6-BA $\left(\mathrm{mg.L}{ }^{-1}\right)$ & IBA $\left(\mathrm{mg} . \mathrm{L}^{-1}\right)$ & $\mathrm{GA}_{3}\left(\mathrm{mg} . \mathrm{L}^{-1}\right)$ \\
\hline ME1 & 0.00 & 0.20 & 1.00 \\
ME 2 & 0.10 & 0.20 & 1.00 \\
ME 3 & 0.20 & 0.20 & 1.00 \\
ME 4 & 0.30 & 0.20 & 1.00 \\
ME 5 & 0.40 & 0.20 & 1.00 \\
ME 6 & 0.50 & 0.20 & 1.00 \\
ME 7 & 0.20 & 0.00 & 1.00 \\
ME 8 & 0.20 & 0.05 & 1.00 \\
ME 9 & 0.20 & 0.10 & 1.00 \\
ME & 0.20 & 0.15 & 1.00 \\
10 & & & \\
ME & 0.20 & 0.25 & 1.00 \\
11 & & 0.20 & 0.00 \\
ME & 0.20 & 0.20 & 0.50 \\
12 & & 0.20 & 1.50 \\
ME & 0.20 & 0.20 & 2.00 \\
13 & 0.20 & 0.20 & 2.50 \\
ME14 & 0.20 & \\
ME15 & 0.20 & & \\
ME16 & 0.20 & &
\end{tabular}

2.4 Effect of different concentrations of hormone and sucrose on rooting

Adventitious buds rooting media were based on $1 / 2 \mathrm{MS}$ medium added different concentration of NAA, IBA and sucrose (Table3). For optimum adventitious bud of embryonic tip rooting media, the replications of the treatments were done two

Table 3.Media of adventitious buds rooting treatment

\begin{tabular}{llll}
\multicolumn{5}{l}{ Table 3. Media of adventitious buds rooting treatment } \\
\hline Code & NAA $\left(\mathrm{mg} . \mathrm{L}^{-1}\right)$ & IBA $\left(\mathrm{mg} . \mathrm{L}^{-1}\right)$ & sucrose $\left(\mathrm{g} . \mathrm{L}^{-1}\right)$ \\
\hline MR1 & 0.50 & 0.00 & 30.00 \\
MR2 & 1.00 & 0.00 & 30.00 \\
MR3 & 1.50 & 0.00 & 30.00 \\
MR4 & 2.00 & 0.00 & 30.00 \\
MR5 & 2.50 & 0.00 & 30.00 \\
MR6 & 0.00 & 0.50 & 30.00 \\
MR7 & 0.00 & 1.00 & 30.00 \\
MR8 & 0.00 & 1.50 & 30.00 \\
MR9 & 0.00 & 2.00 & 30.00 \\
MR10 & 0.00 & 2.50 & 30.00 \\
MR11 & 0.00 & 2.00 & 10.00 \\
MR12 & 0.00 & 2.00 & 20.00 \\
MR13 & 0.00 & 2.00 & 40.00 \\
MR14 & 0.00 & 2.00 & 50.00 \\
\hline
\end{tabular}

times with five repetitions. Each repetition included three explants. After 20d, average rooting rate of adventitious buds was analyzed by one-way ANOVA and LSD ad hoc test with software SPSS16.0.

2.5 Transplanting seedlings 
When the seedlings grow out a taproot, two or more fibrous roots, opened the culture bottles and added some water into the bottles. After 3d, cleaned the media on the roots and transplanted the seedlings into loam(Fig.1e).

\section{EXPERIMENTAL RESULT DISCUSSION}

AND

\subsection{Adventitious bud embryonic tip induction}

\subsubsection{Effect of different seeds soaking time}

With the extension of soaking time, the induction rate of the adventitious buds rised( Fig.1a, Fig. 2). When soaking time was $6 \mathrm{~h}$, the average induction rate was $50 \%$. When soaking time was $12 \mathrm{~h}$, the average induction rate was $85 \%$. When the soaking time was more than $12 \mathrm{~h}$, the average induction rate became low. The average induction rate of adventitious buds was analysed with SPSS16.0 software one-way ANOVA and LSD ad hoc test, the result showed at the different soaking time, the differences of induction rate of adventitious buds were striking $(\mathrm{p}<0.05)$. When soaking time was $12 \mathrm{~h}$, the average induction rate of adventitious buds was the highest. Wang ping ${ }^{[19]}$ used Heinong48 as materials, the result showed that seeds soaking time more than $36 \mathrm{~h}$, the adventitious buds induction rate was the highest, which differ from the results of our experiment, probably because we used JiNong 18 soybean seeds as materials, the varieties of soybeans cause the different.

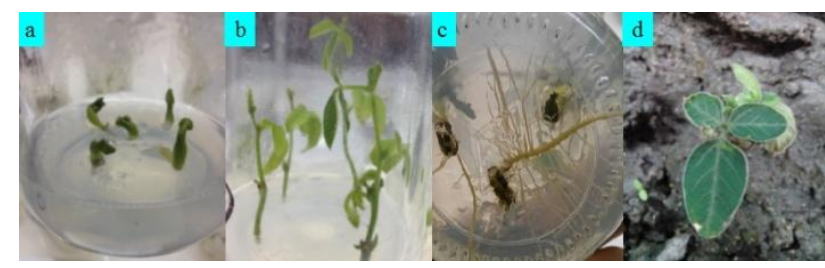

a. Inducted adventitious buds; b. Elongated adventitious buds; c. Inducted roots;

d. Soybean tissue culture seedling

Fig.1 A robust soybean embryonic tip regeneration system

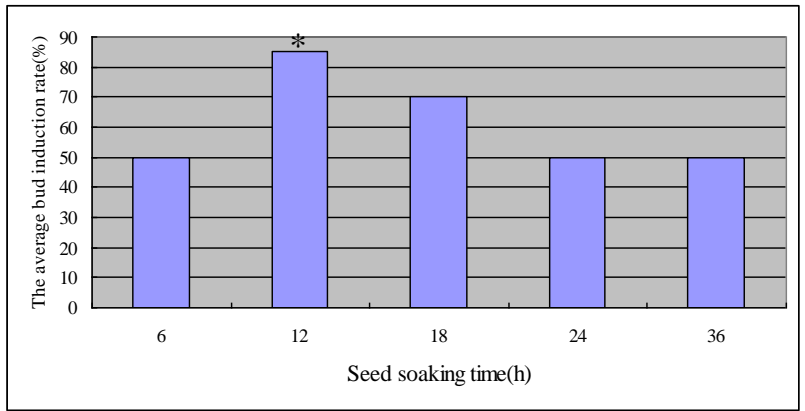

annotation: $*: \mathrm{p}<0.05$

Fig.2 Adventitious bud induction rate under different seeds soaking time

\subsubsection{Effect of different concentrations of 6-BA}

Robust adventitious buds quickly developed in induction media within 10d (Fig.1b, Fig.3), adventitious buds induction rate was the highest on medium MI3(85.0\%) followed by MI2(60\%) and MI4(60\%). The average induction rate of adventitious buds was analyzed by one-way ANOVA and LSD ad hoc test with software SPSS 16.0. The result showed that additional 6-BA in induction medium have a significant different effects on induction rate of adventitious buds ( $p$ $<0.05)$.

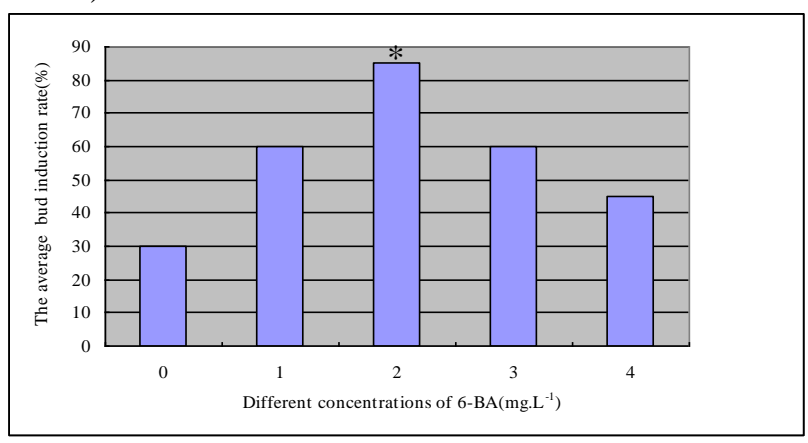

annotation: * : p<0.05

Fig.3 Adventitious bud induction rate ofdifferent concentrations of 6-BA

3.2 Effect of different concentrations of hormone on elongation

When inducted adventitious buds were subcultured on adventitious buds elongation medium, they elongated within $20 \mathrm{~d}$ (Fig.1c, Fig.4-6). The average elongation rate was analyzed by one-way ANOVA and LSD ad hoc test with software SPSS16.0. The result showed that additional 6-BA in adventitious buds elongation medium have not a significant impact on the average elongation rate of adventitious buds ( $\mathrm{p}=0.074864>0.05$ ). The different concentration of additional IBA ( 
$\mathrm{p}=0.031992<0.05)$ and $\mathrm{GA}_{3}(\mathrm{p}=0.001535<$ $0.05)$ were striking.

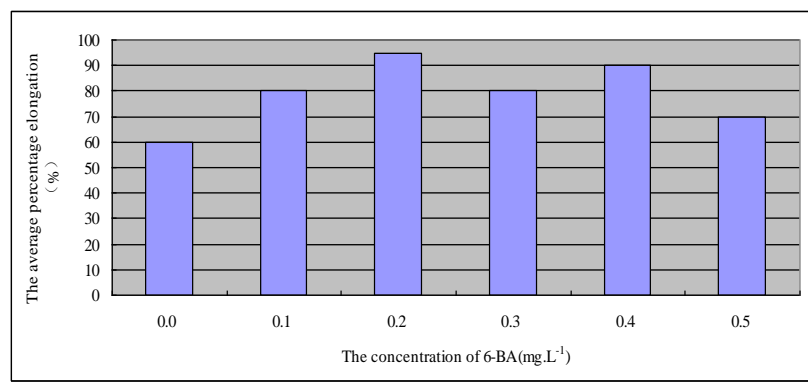

Fig.4 Elongation rate of different concentrations of6BA

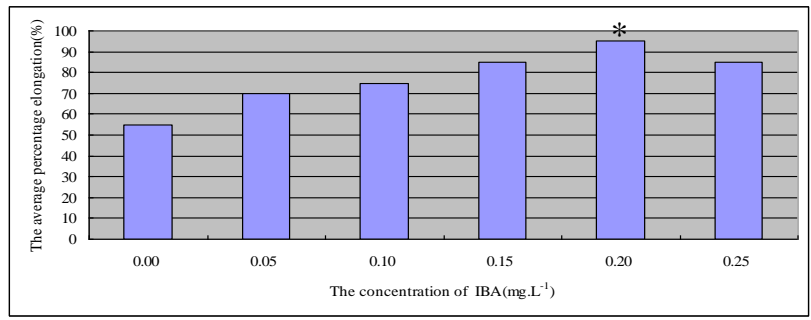

annotation: * : p $<0.05$

Fig.5 Elongation rate of different concentrations ofIBA

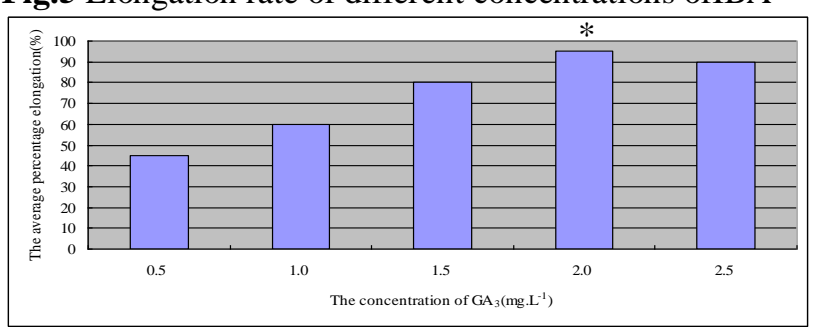

annotation: $*: \mathrm{p}<0.05$

Fig.6 Elongation rate of different concentrations ofGA $\mathrm{A}_{3}$

3.3 Effect of different concentrations of hormone and sucrose on adventitious buds rooting

Adventitious buds were sub-cultured on rooting medium. The experiments proved that NAA and IBAhave thecapability to induce roots. Roots started to grow from 20d (Fig.1d, Fig.7-9). The average rooting rate was analyzed by one-way ANOVA and LSD ad hoc test with software SPSS16.0. Theresult showed that additional NAA or IBA in induction rooting medium have a significant impact on average rooting rate $(\mathrm{p}<0.05)$, and additional sucrose have not a significant $\operatorname{impact}(\mathrm{p}>0.05)$.

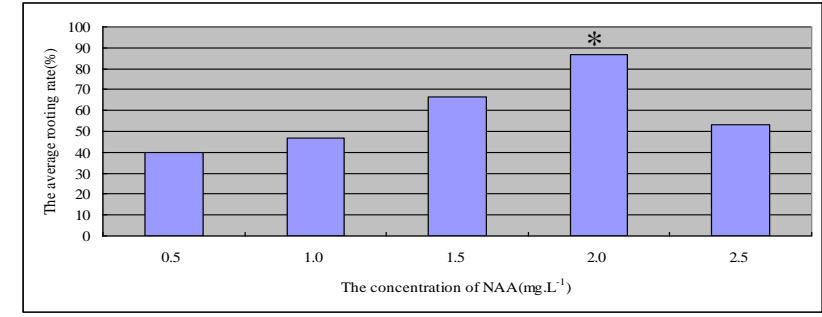

annotation: * : p<0.05

Fig.7 The rooting rate in different concentrations of NAA

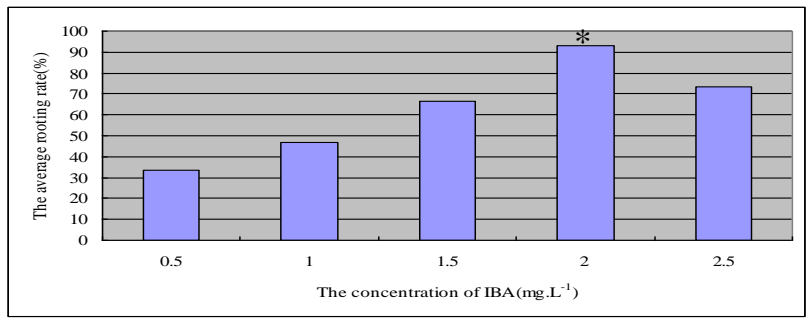

annotation: $*: \mathrm{p}<0.05$

Fig.8 The rooting rate in different concentrations of IBA

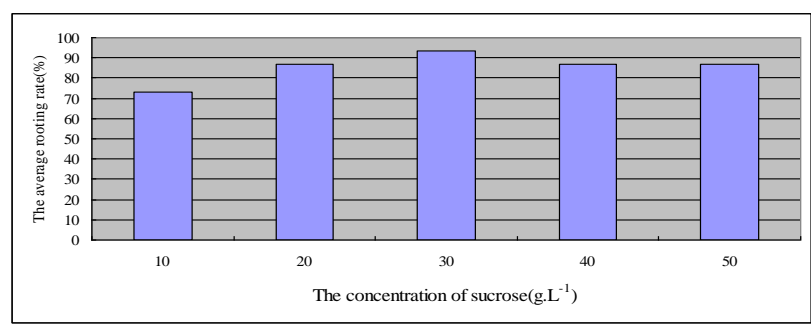

Fig.9 The rooting rate in different concentrations of sucrose

\section{CONCLUSION}

In our experiments, Jinong 18 embryonic tips were used as explants to study various factors of adventitious buds induction, elongation and rooting.

By analysis, we get the conclusions that in the adventitious buds induction stage, both additional 6-BA and seeds soaking time have significant effects. The optimal seeds soaking time is $12 \mathrm{~h}$, and the optimal concentration of $6-\mathrm{BA}$ is $2.0 \mathrm{mg} \cdot \mathrm{L}^{-1}$.

In the adventitious buds elongation stage, additional 6-BA doesn't have significant effects. The concentration of IBA or $\mathrm{GA}_{3}$ in adventitious buds elongation medium has significant effects on average elongation rate. The optimal concentration of IBA is $0.2 \mathrm{mg} \cdot \mathrm{L}^{-1}$. The optimal concentration of $\mathrm{GA}_{3}$ is $2.0 \mathrm{mg} \cdot \mathrm{L}^{-1}$.

In the adventitious buds induction rooting stage, additional sucrose doesn't have significant effect. The concentration of NAA or IBA in induction 
rooting medium have significant effects on average rooting rate. The both optimal concentrations of IBA and $\mathrm{GA}_{3}$ are $2.0 \mathrm{mg} \cdot \mathrm{L}^{-1}$. But additional IBA medium's average rooting rate $(93.34 \%)$ is higher than additional NAA $(86.68 \%)$, so additional IBA on induction rooting is more appropriate.

\section{ACKNOWLEDGMENTS}

This research was supported by The National Natural Science Fund of China(31571689), The Eduation Department of Jilin Province fund of China(2015-191), Jilin Provincial Science \& Technology fund of China(20140204021NY), Jilin Agricultural University initial Scientific Research fund(201242) and student innovation fund (2016).

\section{REFERENCES}

[1] Yan Fan,Sun Xin,Zhai Ying,et al. Effect of Different 6-BA Concentration and Genotypes on Shoots Induced from Embryonic Tips[J].Soybean science, 2011,30(1):19 32

[2] Zhang Dong-xu,Zhang Jie,Shang Lei,et al. Study on the regenration system of soybean embyronic tips[J].Journal of agricultural university of hebei,2008,31(4):7

[3] Cheng $\mathrm{T}$ Y,Saka H,Voqiu-Dinh TH.Plant regeneration from soybean cotyledonary node segments in culture[J].Plant Science Letters, 1980,19: 91 99

[4] Wang Ping,Wang Jun-jun,Shang De-hu,et al.Effect of Induce Factors on Multiple Bud Formation of Cotyledonary Node in Soybean[J].Journal of Jilin Agricultural Sciences,2001,26(6):20 23

[5] Yuan Ying,Liu Depu,Zheng Peihe,et al.Study on Plant Regenerat-ion from Soybean Culture [J]. Soybean science,2001,20(1):9 13

[6] [6]YANG Quan,WANG Yue-yue,LIU Yanguang,et al. Study on Optimization of Soybean Cotyledonary Node Genetic Transformation System and the Transformation of Resistance Gene AtNHX5[J]. Soybean Science,2015,34(2):205 211

[7] Kou Kun,Liu Li-jun,Qu Shan-shan,et al. Improvement of Regen-eration System in New Soybean Line Heinong56 [J]. Soybean Science,2009,28(3): 400 403

[8] Liu Siyan,Gao Wei,Xia Haifeng,et al. Establishment of the Cotyledon Node Regeneration System[J]. Crops, 2013,6:42 45

[9] Liu Hai-Kun,Wei Zhi-ming.Transgenic Soybean Obtained with Agrobacterium tu mefaciensmediated Transformation of Embryonic Tip of Soybean Mature Seeds[J]. Journal of Plant Physiology and Molecular Biology,2004,30(6):631 636

[10] XU Nuo, ZHANG Jun , WANG Pi-wu.Effect of 6-BA and IBA Concentration on Shoots Induction from Embryonic Tips of Four Soybean Genotypes[J].Soybean

Science,2012,31(4):678 679,684
[11] Song Bo,Zhang Xing-guo,Su Cheng-gang,et al Establishment of an Embryonic Tip Regeneration System for Soybeans (Glycine max Merr .) [J].Journal of Southwest China Normal University (Natural Science Edition),2010,35(1):128 131

[12] Cheng Lin-hai,Sun Yi,Liu Shao-xiang,et al.Study on the regeneration of variouse explants of soybean[J].Chinese journal of oil crop sciences, 1998,20(2):24 25

[13] He En-ming, Qi Xiang-jun, Chen Xiu-qing,et al. Callus Induction and Culture of Soybean[J]. Journal of shaanxi University of Science\& Technology,2005,23(5):29 31

[14] Ji Feng-Yuan,Wang Ge-Liang and $\mathrm{Xu}$ YiNong.The Effects Antioxidants on the Transient Expression of GUS Gene in Soybean Hypocotyls Mediated By Agrobacterium Tumefaciens[J].Journal of Plant Ecology, 2006,30(2):330 334

[15] Wei $\mathrm{Z} \mathrm{M}, \mathrm{Xu} \mathrm{Z}$ H.Plant regeneration from protoplasts soybean (Glycine max L.)[J]. Plant Cell Reports, 1988, 7:348 351

[16] Ma Xiao-hong, Yao Lu-ming, Wu Tian-long. High Frequency Plant Regeneration from Whole Cotyledonary Node Explants and Comparison with Cotyledonary Node and Embryonic Tip Regeneration System in Soybean[ Glycinemax(L.) Merrill] [J]. Soybean Science, 2008,27(3):273 278

[17] Meurer C A,Dinkins R D,Collins G B.Factors affecting soybean cotyledonary node transformation[J]. Plant Cell Reports, 1998, 18:180 186

[18] Donaldson P A, Simmonds D H. Susceptibility to Agrobacterium tumefaeiens and cotyledonary node transformation in short-season soybean[J]. Plant Cell Reports, 2000, 19:478 484

[19] Wang Ping, ZhangYanjun, GuanJuanjuan, et al.Induction of Adventitious Buds from Embryonic Tip in Soybean[J].Crops, 2011(1):7 19PAN Guang-bin, CHEN Guang-ju. Design of Indirectly Measuring Model Based on SVD [J]. Journal of Electronic Mearurement and Instrument, 2006,20(5):60-62. 